\title{
The induction of recombinant protein bodies in different subcellular compartments reveals a cryptic plastid-targeting signal in the 27-kDa $\gamma$-zein sequence
}

\author{
Anna Hofbauer ${ }^{1 \dagger}$, Jenny Peters ${ }^{1 \dagger}$, Elsa Arcalis ${ }^{1}$, Thomas Rademacher $^{2}$, Johannes Lampel ${ }^{1}$, François Eudes ${ }^{3}$, \\ Alessandro Vitale ${ }^{4}$ and Eva Stoger ${ }^{1 *}$
}

${ }^{1}$ Department of Applied Genetics and Cell Biology, University of Natural Resources and Life Sciences, Vienna, Austria

2 Institute of Molecular Biotechnology, RWTH Aachen University, Aachen, Germany

${ }^{3}$ Agriculture and Agri-Food Canada, Lethbridge, AB, Canada

${ }^{4}$ Institute of Agricultural Biology and Biotechnology, National Research Council (CNR), Milan, Italy

Edited by:

Giuseppe Dionisio, Aarhus University, Denmark

\section{Reviewed by:}

Martine Gonneau, Institut National de la Recherche Agronomique, France Anneli Marjut Ritala, VTT Technical Research Center, Finland

*Correspondence:

Eva Stoger, University of Natural Resources and Life Sciences,

Muthgasse 18, Vienna 1190, Austria e-mail: eva.stoger@boku.ac.at

${ }^{\dagger}$ Anna Hofbauer and Jenny Peters have contributed equally to this work.
Naturally occurring storage proteins such as zeins are used as fusion partners for recombinant proteins because they induce the formation of ectopic storage organelles known as protein bodies (PBs) where the proteins are stabilized by intermolecular interactions and the formation of disulfide bonds. Endogenous PBs are derived from the endoplasmic reticulum (ER). Here, we have used different targeting sequences to determine whether ectopic PBs composed of the N-terminal portion of mature $27 \mathrm{kDa} \gamma$-zein added to a fluorescent protein could be induced to form elsewhere in the cell. The addition of a transit peptide for targeting to plastids causes PB formation in the stroma, whereas in the absence of any added targeting sequence PBs were typically associated with the plastid envelope, revealing the presence of a cryptic plastid-targeting signal within the $\gamma$-zein cysteine-rich domain. The subcellular localization of the PBs influences their morphology and the solubility of the stored recombinant fusion protein. Our results indicate that the biogenesis and budding of PBs does not require ER-specific factors and therefore, confirm that $\gamma$-zein is a versatile fusion partner for recombinant proteins offering unique opportunities for the accumulation and bioencapsulation of recombinant proteins in different subcellular compartments.

Keywords: protein bodies, molecular farming, subcellular targeting, intermembrane space, plastid import, recombinant protein

\section{INTRODUCTION}

Plants are versatile platforms for the production of recombinant pharmaceutical proteins and peptides because they are safe, scalable, and allow long-term protein storage (Ma et al., 2013; Rybicki et al., 2013; Stoger et al., 2014). It is usually advantageous if the protein accumulates at a high concentration and remains stable after harvest, particularly in the case of antigens, antibodies, and enzymes produced for oral application, where doses must be up to 1000 -fold higher than parental formulations to ensure that sufficient amounts of the protein survive proteolytic digestion.

The stability of recombinant proteins upon administration can be increased by in vitro encapsulation, which usually involves spray or freeze drying methods and mixing with plant-derived components such as cereal storage proteins (Zhong and Jin, 2009; Zou and $\mathrm{Gu}, 2013$ ). Prolamin-type storage proteins (e.g., maize zeins) are often used for this purpose because their film-building properties allow them to form coatings and microparticle formulations, and they also have GRAS food use status (generally recognized as safe by the U.S. Food and Drug Administration) and high resistance to digestion (Liu et al., 2005; Wang et al., 2011; Lau et al., 2013). When using plants as production hosts, it is therefore appealing to attempt microencapsulation in vivo by directly incorporating the recombinant protein into protein storage bodies (Hofbauer and Stoger, 2013). This is the typical strategy used with seed-based production systems, where the recombinant protein is often targeted so that it accumulates in prolamin-containing storage organelles. Several studies have shown that recombinant proteins incorporated in storage organelles are protected from proteolytic digestion in simulated gastric fluids and are more effective following oral delivery, indicating the potential of this strategy for the bioencapsulation of recombinant pharmaceutical proteins and their efficient mucosal delivery (Chikwamba et al., 2003; Nochi et al., 2007; Takagi et al., 2010; Wakasa et al., 2013).

Protein bodies (PBs) form naturally in the endoplasmic reticulum (ER) of developing cereal endosperm cells, but the expression of recombinant proteins fused to assembly sequences can induce analogous organelles in tissues such as leaves, which usually lack these structures. Sequences that can induce the formation of ectopic PBs and thus increase the yield and stability of recombinant proteins include those derived from cereal prolamins, synthetic elastin-like peptides, and fungal hydrophobins (Floss et al., 2008; Conley et al., 2009; Torrent et al., 2009b; Gutierrez et al., 2013; Shigemitsu et al., 2013).

One of the most widely used assembly sequences is found near the $\mathrm{N}$-terminus of the $27 \mathrm{kDa} \gamma$-zein, a member of the major 
prolamin-type storage protein family in maize (Shewry and Halford, 2002). About 15 zein genes (divided into $\alpha$-, $\beta, \delta$, and $\gamma$-zeins) are expressed at high levels in maize endosperm and condense together in the ER, to from the heteropolymeric PBs that often bud off the ER as distinct round shaped structures that maintain polyribosomes on the cytosolic surface (Lending and Larkins, 1989). The $\gamma$-zeins form the peripheral layer of PBs and play a structural role in their biogenesis (Ludevid et al., 1984). Ectopically expressed zein under the control of the constitutive $35 \mathrm{~S}$ promoter forms ER-resident PBs in vegetative tissues, underscoring its intrinsic compartment-forming properties in the absence of tissue-specific factors (Bagga et al., 1995). The PB-inducing properties of $27 \mathrm{kDa} \gamma$-zein have been causally linked to its $\mathrm{N}$-terminal half, comprising a domain with two Cys residues that follows the signal peptide, a repeated Pro-rich domain forming an amphipathic helix, and a third domain that includes four additional Cys residues (Geli et al., 1994). Mutagenesis of the Cys residues or the amphipathic repeat in fusion proteins or full length $27 \mathrm{kDa}$ $\gamma$-zein indicate that the repeat favors $\mathrm{PB}$ formation, but the Cys residues have a fundamental role (Mainieri et al., 2004; Llop-Tous et al., 2010). Several reports have confirmed that the zein domain appended to the C-terminus of polypeptides with their own signal peptide for translocation in the ER, or an extended version (Zera ${ }^{\circledR}$ ) that includes the zein signal peptide and can thus be placed at the $\mathrm{N}$-terminus, can successfully trigger PB formation and high accumulation when fused to diverse recombinant proteins such as bean phaseolin (Mainieri et al., 2004), enhanced cyan fluorescent protein (Llop-Tous et al., 2010), xylanase (Llop-Tous et al., 2011), DsRed (Joseph et al., 2012), and human papillomavirus (Whitehead et al., 2014) and can be used not only in vegetative plant tissues but also in fungal, insect, and mammalian cells (Torrent et al., 2009a).

The accumulation, folding, and post-translational modification of recombinant proteins depend on their subcellular localization, because the cytosol, the ER, peroxisomes, and the different subcompartments of semiautonomous organelles have specific chemical features and repertoires of protein folding helpers. Targeting strategies must therefore be adapted for each candidate protein, and the addition of signal sequences to direct proteins to different subcellular compartments is often used to optimize production (Hofbauer and Stoger, 2013). Natural storage organelles originate from the endomembrane system and most ectopic PBs have accordingly been derived from the ER, whereas PB induction in other compartments has met with only limited success (Bellucci et al., 2005; De Marchis et al., 2012). Here, we used N-terminal portions of $27 \mathrm{kDa} \gamma$-zein as fusion partners for the visual marker DsRed, and the fusion proteins were directed to different compartments to investigate whether PBs can be encouraged to form outside the endomembrane system. We found that constructs with a plastid-targeting peptide led to the induction of PBs, but that constructs lacking this peptide were nevertheless able to form PBs associated with plastids, revealing the presence of a cryptic plastidtargeting signal within the $\mathrm{PB}$-inducing $\mathrm{N}$-terminal region of the mature $\gamma$-zein. We provide confocal and electron microscopy evidence for the localization of the PBs in different compartments within the plastid and consider the nature of the plastid import pathways.

\section{MATERIALS AND METHODS VECTOR CONSTRUCTS}

All cloning steps were carried out with the binary vector pTRA, a derivative of pPAM (GenBank AY027531). Tetrameric DsRed (Jach et al., 2001) was joined via a (GGGS) 2 linker to residues $4-93$ of the mature $27 \mathrm{kDa} \gamma$-zein protein (lacking the signal peptide) in the same way as described for the PB-forming phaseolin fusion construct zeolin (Mainieri et al., 2004) to generate our basic vector $\Delta$ SP-DsZein. In construct SP-DsZein, a plant codon-optimized signal peptide sequence derived from a murine antibody (SP) was added at the $\mathrm{N}$-terminus to direct the protein into the secretory pathway. In construct TP-DsZein, the 69-amino-acid plastid transit peptide sequence from the barley GBSSI gene (accession no. AF486514) was added instead. A peptide analysis using ChloroP prediction program (http://www.cbs.dtu.dk/services/ChloroP/) identified a potential chloroplast targeting peptide of 43 residues (cTP score of 0.460 ) in the $\mathrm{N}$-terminal part of the mature $27 \mathrm{kDa}$ $\gamma$-zein protein. This sequence comprising residues $51-93$ of the $\gamma$-zein sequence was fused to DsRed via the (GGGS) $)_{2}$ linker to test the predicted function of this peptide. The resulting construct was termed $\triangle$ SP-DsCRS. For the control construct SP-DsHR, we used three repeats of the sequence PPPVRL fused to DsRed. This sequence is similar to the repeat domain of the zein sequence and does not include cysteine residues. $\mathrm{A} \mathrm{His}_{6}$ tag was added to the C-terminus of all constructs as an additional means for detection. The synthetic fusion sequences were inserted into the pTRA-vector between the Tobacco etch virus (TEV) $5^{\prime}$-untranslated region and the Cauliflower mosaic virus (CaMV) $35 \mathrm{~S}$ terminator (Figure 1). The expression construct was thus placed under the control of the CaMV 35S promoter with duplicated transcriptional enhancer.

\section{PLANT MATERIAL}

Tobacco (Nicotiana tabacum cv. SR1) plants were cultivated in soil in growth chambers at $26^{\circ} \mathrm{C}$ and $70 \%$ humidity with a 16$\mathrm{h}$ photoperiod. The TOC-GFP tobacco marker line, expressing a marker that highlights the outer plastid envelope, was kindly provided by Dr. Maureen Hanson, Cornell University, Ithaca, NY, USA (Hanson and Sattarzadeh, 2008) and cultivated under the same conditions.

\section{AGROINFILTRATION OF TOBACCO LEAVES}

Agrobacterium tumefaciens (GV3101) preserved as a glycerol stock was inoculated into $5 \mathrm{ml}$ aliquots of YEB medium containing $25 \mathrm{mg} / \mathrm{l} \mathrm{kanamycin,} 25 \mathrm{mg} / \mathrm{l}$ rifampicillin, and $50 \mathrm{mg} / \mathrm{l}$ carbenicillin, and incubated for 2 days at $28^{\circ} \mathrm{C}$, shaking at $180 \mathrm{rpm}$. The $\mathrm{OD}_{600}$ of the culture was adjusted to $\sim 1.0$ with $2 \times$ infiltration medium (100 g/l sucrose, $3.6 \mathrm{~g} / \mathrm{l}$ glucose, $8.6 \mathrm{~g} / \mathrm{l} \mathrm{MS} \mathrm{salts,} \mathrm{pH} 5.6$ ) and $200 \mu \mathrm{M}$ acetosyringone was added before infiltration into tobacco leaves. The infiltrated leaves were harvested 3-10 days post-infiltration (DPI).

\section{PROTEIN IMMUNOBLOT ANALYSIS}

Infiltrated leaves (7 DPI) were harvested and ground in liquid nitrogen to a fine powder. For each sample, $60 \mathrm{mg}$ leaf powder were extracted in $200 \mu \mathrm{l}$ phosphate buffered saline (PBS, pH 7.4) and shaken for $15 \mathrm{~min}$ at room temperature. After centrifugation at $13,000 \times g$ for $15 \mathrm{~min}$ at room temperature, the supernatant 
A

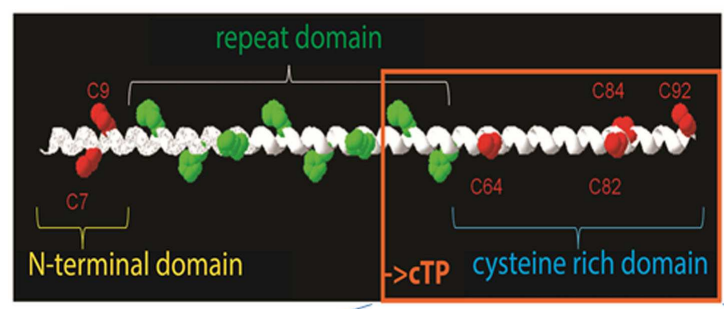

HVPPPVHLPPPPCHYPTQPPRPQPHPQPHPCPCQQPHPSPCQ
B

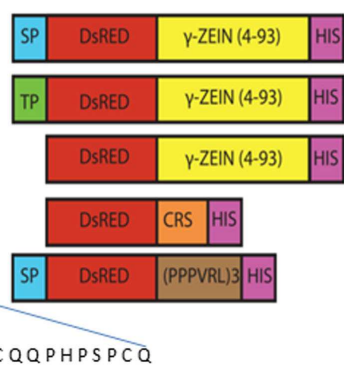

\begin{tabular}{|cc}
\hline Name & $\begin{array}{c}\text { Expected } \\
\text { Targeting }\end{array}$ \\
\hline SP-DsZein & ER \\
\hline TP-DsZein & Plastid \\
DSP-DsZein & Cytosol \\
\hline DSP-DsCRS & Cytosol \\
\hline SP-DSHR & ER \\
\hline
\end{tabular}

(B) Schematic overview of the expression constructs. SP, signal peptide for entry into the ER; TP, plastid-targeting peptide; DsZein, fluorescent marker protein plus $\gamma$-zein(4-93); CRS, cysteine-rich sequence (residues 51-93); HR, hydrophobic repeat sequence (PPPVRL) $)_{3}$ HIS, polyhistidine tag.
(PBS-soluble fraction) was transferred to a fresh tube and the pellet (insoluble fraction) was washed three times with $\mathrm{PBS}$ before re-extraction with buffer $\mathrm{K}(62.5 \mathrm{mM}$ Tris $\mathrm{pH} 7.4,10 \%$ glycine, $5 \%$ 2-mercaptoethanol, $2 \%$ SDS, $8 \mathrm{M}$ urea). The relative quantity of total recombinant protein was estimated by directly homogenizing $60 \mathrm{mg}$ leaf samples in $200 \mu \mathrm{l}$ buffer K, sonicating for $20 \mathrm{~s}$, and centrifuging as above for $10 \mathrm{~s}$. Protein samples were boiled in loading buffer, separated by SDS-PAGE in 12\% acrylamide gels and transferred to a nitrocellulose membrane. DsRed-fusion proteins were detected using an anti-His-tag antibody. The recombinant protein was visualized with an alkaline phosphatase-conjugated goatanti-mouse IgG (Promega, Fitchburg, WI, USA) diluted 1:5000. Images were analyzed using Image Lab v5.1 (Bio-Rad Laboratories, Hercules, CA, USA).

\section{FLUORESCENCE AND ELECTRON MICROSCOPY}

Infiltrated leaves were cut into small pieces with a razor blade. For fluorescence microscopy, the samples were mounted in tap water on a glass slide and DsRed fluorescence was observed under a Leica SP5 confocal laser scanning microscope (CLSM).

For immunolocalization by electron microscopy, the samples were fixed in $4 \%(\mathrm{w} / \mathrm{v})$ paraformaldehyde plus $0.5 \%(\mathrm{v} / \mathrm{v}) \mathrm{glu}-$ taraldehyde in $0.1 \mathrm{M}$ phosphate buffer $(\mathrm{pH} 7.4)$ at $4^{\circ} \mathrm{C}$ overnight, then dehydrated through an ethanol series and polymerized in LR White resin as previously described (Arcalis et al., 2010). Ultra-thin sections were mounted on gold grids, blocked with $5 \%(\mathrm{w} / \mathrm{v})$ bovine serum albumin in $0.1 \mathrm{M}$ phosphate buffer $(\mathrm{pH}$ 7.4) and incubated with the polyclonal Living Colors DsRed antibody. The samples were then incubated with a rabbit-anti-goat IgG linked to $10-\mathrm{nm}$ colloidal gold and stained in $2 \%$ aqueous uranyl acetate.

For ultrastructural studies by electron microscopy, the samples were fixed in and in $2 \%(\mathrm{w} / \mathrm{v})$ paraformaldehyde plus $2.5 \%$ (v/v) glutaraldehyde in $0.1 \mathrm{M}$ phosphate buffer $(\mathrm{pH} 7.4)$ at $4^{\circ} \mathrm{C}$ overnight followed by an additional post-fixing step where samples were incubated in $1 \%(\mathrm{w} / \mathrm{v})$ osmium tetroxide with $0.8 \%$ $(\mathrm{w} / \mathrm{v}) \mathrm{KFeCN}$ in $0.1 \mathrm{M}$ phosphate buffer $(\mathrm{pH}$ 7.4) for $3 \mathrm{~h}$ at room temperature. After dehydration through an acetone series, the samples were polymerized in Spurr epoxy resin as previously described (Arcalis et al., 2010) and observed under a FEI Tecnai $\mathrm{G}^{2}$ transmission electron microscope.

\section{RESULTS}

\section{PROTEIN BODIES CAN FORM OUTSIDE THE ER AND THE COMPARTMENT DETERMINES THEIR MORPHOLOGY AND THE SOLUBILITY OF THE STORED RECOMBINANT PROTEIN}

The N-terminal portion of $\gamma$-zein is necessary for the ability of this protein to form PBs in the ER (Geli et al., 1994). To investigate whether PBs can also be created in other compartments, we designed a fusion protein comprising DsRed and 89 residues of $\gamma$-zein starting from the fourth amino acid after the N-terminal signal peptide cleavage site, hereafter $\gamma$-zein(4-93). This is the same zein fragment that promotes the formation of ER-located PBs when fused at the C-terminus of the vacuolar storage protein phaseolin, in the chimeric protein zeolin (Mainieri et al., 2004). The fragment was the basis for different constructs targeting the fusion protein to the cytosol ( $\Delta$ SP-DsZein), ER (SP-DsZein), and plastids (TP-DsZein). As control, we used a sequence that is similar to the hydrophobic domain in $\gamma$-zein(4-93) but lacks any cysteine residues, and thus lacks the ability to form PBs (SPDsHR). An overview of these constructs is provided in Figure 1. $\mathrm{PB}$ formation was induced with all constructs except the negative control (Figure 2A). A comparison of PB biogenesis and growth in the different compartments indicated that PBs induced by the SP-DsZein and TP-DsZein constructs were limited to a size of 1$1.5 \mu \mathrm{m}$, which was achieved $\sim 4 \mathrm{DPI}$, and showed a homogeneous size distribution. In contrast, the PBs induced by $\triangle$ SP-DsZein had an average diameter of $\sim 3 \mu \mathrm{m}$ and were heterogeneous in terms of size and growth rate (Figure 2B).

Infiltrated leaves were harvested 7 DPI and the solubility of the four DsRed-fusion proteins was determined by extraction in PBS and re-extraction of the pellet under strong reducing conditions. Following infiltration with SP-DsZein and TP-DsZein, most of the DsRed-fusion protein was only soluble after re-extraction. However, infiltration with $\triangle$ SP-DsZein yielded a minor soluble fraction, and following infiltration with the control construct 

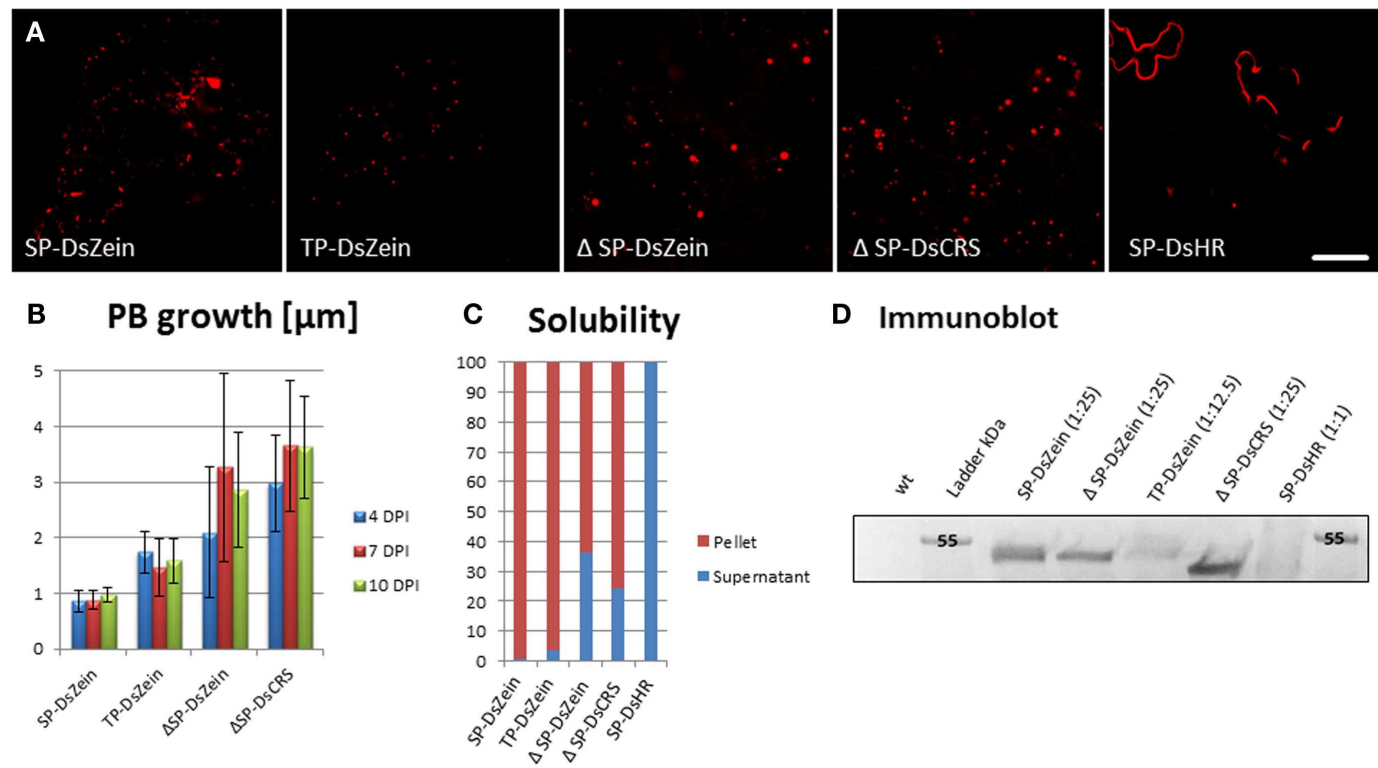

D Immunoblot

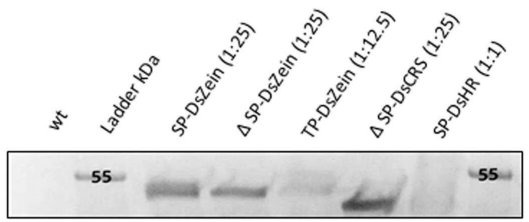

FIGURE 2 | (A) Overview of the fluorescent signal distribution following transient expression with the constructs shown in Figure 1. (B) Protein body growth and size distribution after infiltration. Bars represent the standard error; $n=25$. (C) Solubility of the recombinant fusion proteins. Leaf samples

were extracted in PBS (soluble fraction) and pellets were then re-extracted under strong reducing conditions. (D) Immunoblot analysis of total protein extracts from leaf samples. Antiserum against the HIS-tag was used to detect the recombinant protein.

SP-DsHR all of the recombinant fusion protein was soluble in PBS, as expected (Figure 2C).

The infiltrated leaves were also homogenized directly in strong reducing buffer. The total leaf extracts were then analyzed by immunoblot to confirm the molecular masses of the proteins and to compare the relative amounts of recombinant fusion protein obtained with the different constructs (Figure 2D). All PB-inducing fusion proteins led to higher yields of the chimeric protein than the control construct for secretion, SPDsHR (Figure 2D). A further control lacking both the signal peptide and the cysteine-rich sequence, $\triangle \mathrm{SP}$-DsHR, resulted in even lower expression in N. tabacum and was not detectable by immunoblot or fluorescence microscopy (data not shown). Infiltration of this construct into $N$. benthamiana led to slightly higher expression levels and microscopy analysis confirmed that this construct caused cytosolic distribution of the fusion protein without induction of PBs (Figure S1 in Supplementary Material).

\section{SP-DsZein AND TP-DsZein INDUCE PROTEIN BODIES IN THE ER AND PLASTIDS, RESPECTIVELY}

The formation of PBs was investigated by confocal microscopy 4-15 DPI. Construct SP-DsZein induced the formation of spherical bodies with a tendency to form clusters (Figure 3A) and electron microscopy confirmed the presence of PBs in the cytoplasm (Figure 3B), surrounded by a ribosome-studded membrane, indicating they had originated from the ER (Figure 3C). Construct TP-DsZein also induced the formation of spherical PBs but their distribution differed significantly from those induced by SP-DsZein. Confocal microscopy clearly revealed the formation of fluorescent $\mathrm{PBs}$ in the stroma (Figure 4). Most plastids contained a single $\mathrm{PB}$ in the stroma (Figure 4A). PBs formed by SP-DsZein

are, like natural PBs of maize endosperm, in close contact to the inner face of the ER membrane (Figure 3B). Those formed by TP-DsZein are only in part, but not completely, in contact with thylakoids.

\section{THE $\triangle$ SP-DsZein CONSTRUCT INDUCES PROTEIN BODIES ASSOCIATED WITH THE PLASTID ENVELOPE}

The transient expression of a construct lacking an N-terminal signal sequence ( $\Delta$ SP-DsZein) induced the formation of large PBs $(\sim 3 \mu \mathrm{m}$ in diameter) that appeared to be localized mostly in close association with the plastids (Figure 5A). Some plastids were characterized by an irregular red fluorescent periphery, suggesting that smaller PBs were budding from the plastid membrane (Figure 5B). Indeed, electron microscopy revealed several PBs budding from the plastid surface (Figures 5C,D). Interestingly, tubular thylakoids [typically a sign of plastid stress; (Monselise et al., 1984)] were observed in the vicinity of these budding sites (Figures 5C,D). Detailed images revealed that recombinant protein accumulating at the plastid periphery was actually confined by two membranes, one enclosing the $\mathrm{PB}$ on the outside and the other defining the stromal border, indicating that the recombinant protein is localized in the intermembrane space (IMS), between the inner and outer membranes of the plastid envelope (Figure 5D). On several instances the PB protrudes from the chloroplast, suggesting a process of budding from the intermembrane space (Figures 5C,D). Lipid staining revealed that the budding PBs are enclosed in a lipid membrane derived from the outer membrane of the plastid envelope (Figure 5D).

To confirm these results, the $\Delta$ SP-DsZein construct was transiently expressed in the leaves of a TOC-GFP tobacco marker line, which expresses a fluorescent marker that identifies the 


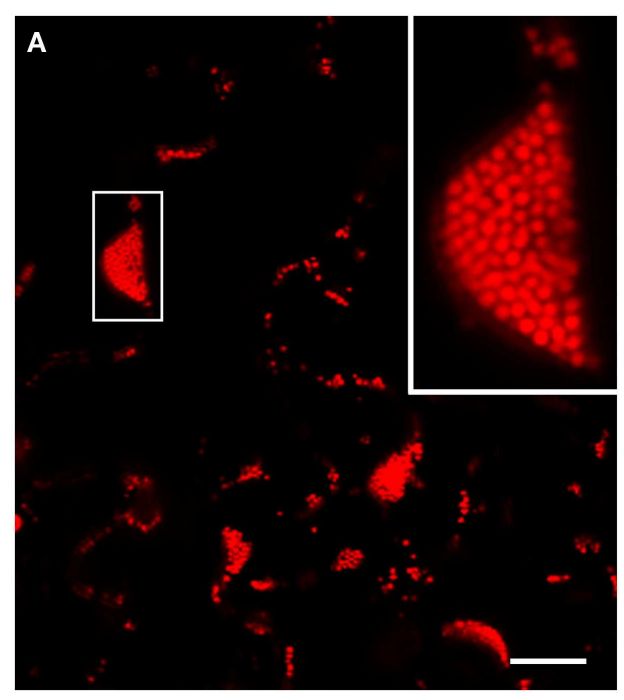

FIGURE 3 | Protein body formation in the ER (SP). (A) Confocal laser scanning microscopy. Infiltrated leaf tissue overview, showing abundant protein bodies. The inset shows an enlargement of the outlined protein body cluster. (B,C) Electron microscopy. Spherical
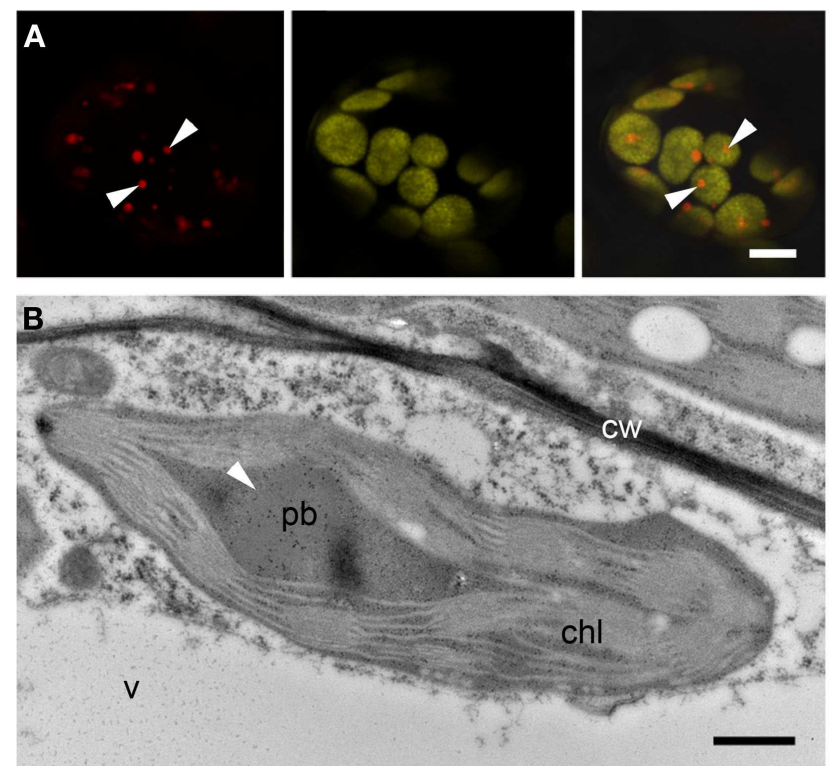

FIGURE 4 | Protein body formation in the plastids (TP). (A) Confocal laser scanning microscopy. Protein bodies (arrowheads) within the plastids. Left: DsRed fluorescence, middle: plastid autofluorescence, right: merged channels. Note that in most cases one protein body can be observed per plastid. (B) Immunoelectron microscopy. Protein body (pb, arrowhead) within the stroma of the plastid (chl). Mitochondria $(\mathrm{m})$. Bars $=5 \mu \mathrm{m}(\mathbf{A})$ or $0.5 \mu \mathrm{m}$ (B)

outer plastid membrane (Hanson and Sattarzadeh, 2008). CLSM allowed the collection of $\mathrm{z}$-series images from plastids and associated red fluorescent $\mathrm{PBs}$, confirming that the PBs were covered by the outer plastid membrane (Figure 5E).
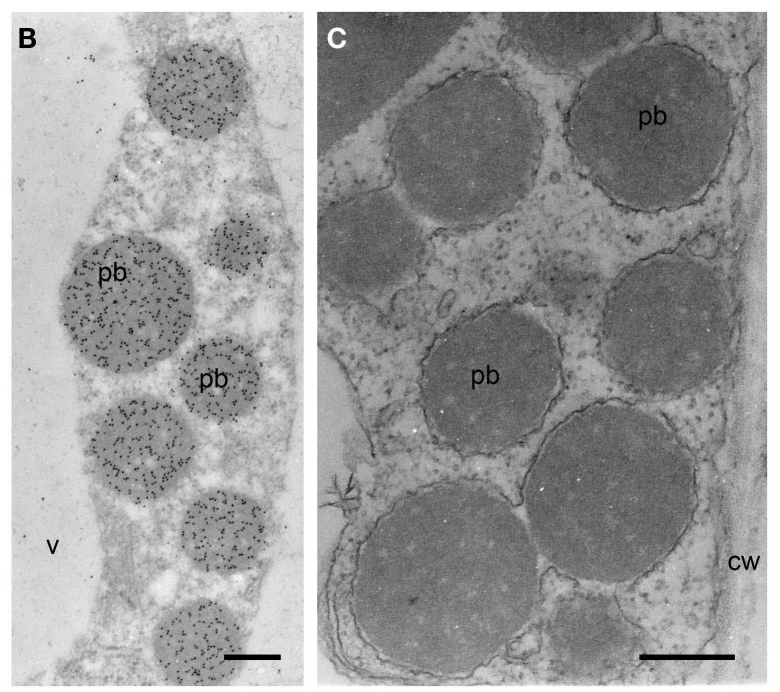

protein bodies (pb) showing abundant, specific labeling for DsRed (B), surrounded by a ribosome-studded membrane [(C), arrowhead] Cell wall (cw), vacuole (v). (C) Lipid staining. Bars $=25 \mu \mathrm{m}$ (A) or $0.5 \mu \mathrm{m}(\mathbf{B}, \mathbf{C})$.

\section{THE CYSTEINE-RICH SEQUENCE OF $\gamma$-zein CONTAINS A CRYPTIC PLASTID-TARGETING SIGNAL}

To narrow down the region in the $\mathrm{N}$-terminal portion of $\gamma$-zein responsible for the de novo formation of $\mathrm{PBs}$ in the plastid envelope, we designed a further construct containing DsRed and the distal part of the $\gamma$-zein (4-93) fragment described above, hereafter $\gamma$-zein(51-93). The construct was named $\Delta$ SP-DsCRS because the short zein fragment retains the four Cys residues that follow the repeated region and is therefore a cysteine-rich sequence (CRS).

This construct behaved similarly to $\triangle$ SP-DsZein, forming large and heterogeneous $\mathrm{PBs}$ that grew at different rates and in some cases reached up to $5 \mu \mathrm{m}$ in diameter (Figures $2 \mathrm{~A}, \mathbf{B}$ and $\mathbf{6 A - C}$ ). As with $\triangle$ SP-DsZein, a relevant fraction of the DsRed-fusion protein was soluble in the absence of reducing agent (Figure 2C). By 15 DPI, large and irregular PBs were observed, which appeared to be budding from the plastid surface (Figures 6C,D). These large fluorescent $\mathrm{PBs}$ could even be observed by bright field microscopy (Figure 6A). Electron microscopy revealed frequent associations between these PBs and the plastids, as described for $\triangle$ SP-DsZein, and smaller PBs could again be observed budding from the plastid envelope (Figure 6D). Occasionally, the fusion protein is distributed as electron dense material within the intermembrane space without forming a spherical PB (Figure 6E). Transient expression in the leaves of TOC-GFP tobacco plants again confirmed that the PBs were covered by the outer plastid membrane (Figure 6F).

\section{DISCUSSION}

\section{PB FORMATION IN THE CHLOROPLAST STROMA}

Protein bodies have been induced mainly as derivatives of the endomembrane system, whereas there have been few attempts to induce PBs in the cytosol or plastids (Bellucci et al., 2005, 2007; De Marchis et al., 2011). We used DsRed fused to different stretches of 

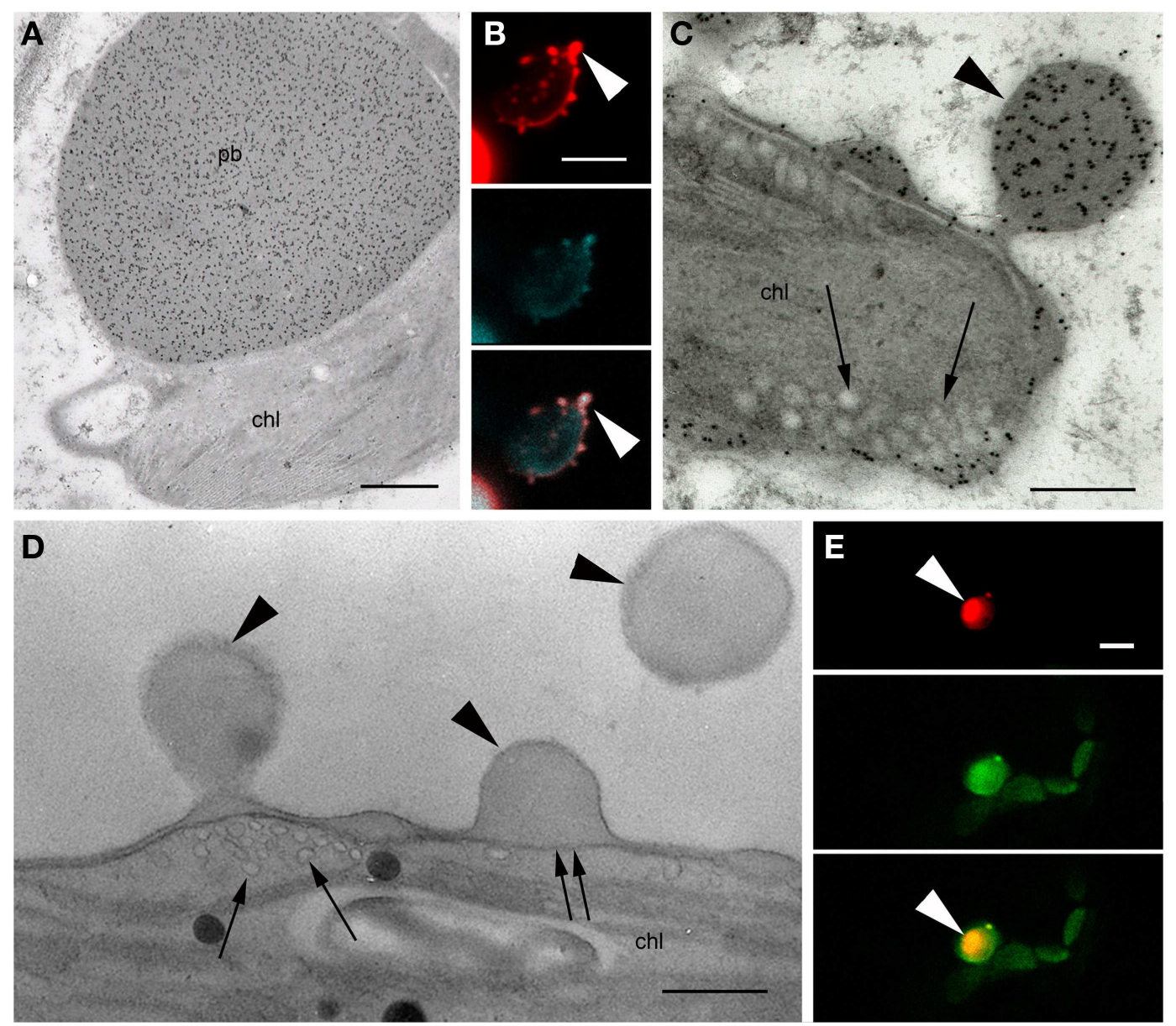

\section{FIGURE $\mathbf{5}$ | Protein bodies induced by $\Delta$ SP-DsZein}

(A) Immunoelectron microscopy. Gold particles decorating a protein body (pb) in close association with a plastid (chl). (B) CLSM image showing the accumulation of fluorescent fusion protein in the periphery of a plastid, as well as several budding sites (arrowhead). DsRed fluorescence (top), autofluorescence of plastids (middle), and merged channels (bottom) are shown. (C) Immunoelectron microscopy, localization of DsRed. Abundant gold probes are visible in the periphery of a plastid, showing a budding protein body (arrowhead). Note the tubular thylakoids in the vicinity of the budding site (arrows). (D) Detection of unsaturated lipids, by electron microscopy. Budding protein bodies are confined by the outer membrane (arrowheads) and the inner envelope membrane (double arrow) of the plastid. Tubular thylakoids can be observed at the budding site (arrows). (E) CLSM image showing red fluorescent protein bodies enclosed by the outer plastid envelope membrane highlighted with a TOC-GFP membrane marker (arrowheads). DsRED (top), GFP (middle) and merged channels (bottom) are shown. Bars $=0.5 \mu \mathrm{m}(\mathbf{A}, \mathbf{D}), 5 \mu \mathrm{m}(\mathbf{B}, \mathbf{E})$, or $0.25 \mu \mathrm{m}$ (C) the $\mathrm{N}$-terminal portion of mature $\gamma$-zein and designed constructs targeting the cytosol, ER, and plastids. All three constructs were able to induce PBs, however, a control construct containing an amphipathic repeat domain similar to the zein sequence but lacking the cysteine-rich element was unable to form PBs, as expected from previous mutagenesis experiments performed on $\gamma$-zein or fusions derived from it (Pompa and Vitale, 2006).

It was somewhat surprising that the TP-DsZein construct was able to induce the formation of DsRed PBs within the plastids, whereas the phaseolin-zein fusion protein zeolin was unable to form PBs in the plastid and instead accumulated as monomers without disulfide bonds (Bellucci et al., 2007; De Marchis et al., 2011). This discrepancy may reflect the different expression strategies - zeolin was produced by plastid transformation followed by direct accumulation within the plastid, whereas TP-DsZein was produced by transient expression in the nucleus followed by targeting to the plastid as a post-translational event. Alternatively, the different intrinsic properties of the proteins may be relevant - DsRed is a well-established marker which is known to accumulate in plastids whereas phaseolin might interfere with protein homeostasis in the plastid or may not reach the minimum concentration necessary for PB formation (Gutierrez et al., 2013). Several post-translational modifications are known to take place in the stroma of tobacco plastids (Glenz et al., 2006) including the formation of disulfide bonds (Bally et al., 2008). A number of previous reports describe the expression, in the plastid stroma, of biologically functional proteins that require intra-chain and/or inter-chain disulfide bonds for their activity (Staub et al., 2000; Daniell et al., 2001; Lakshmi et al., 2013). 

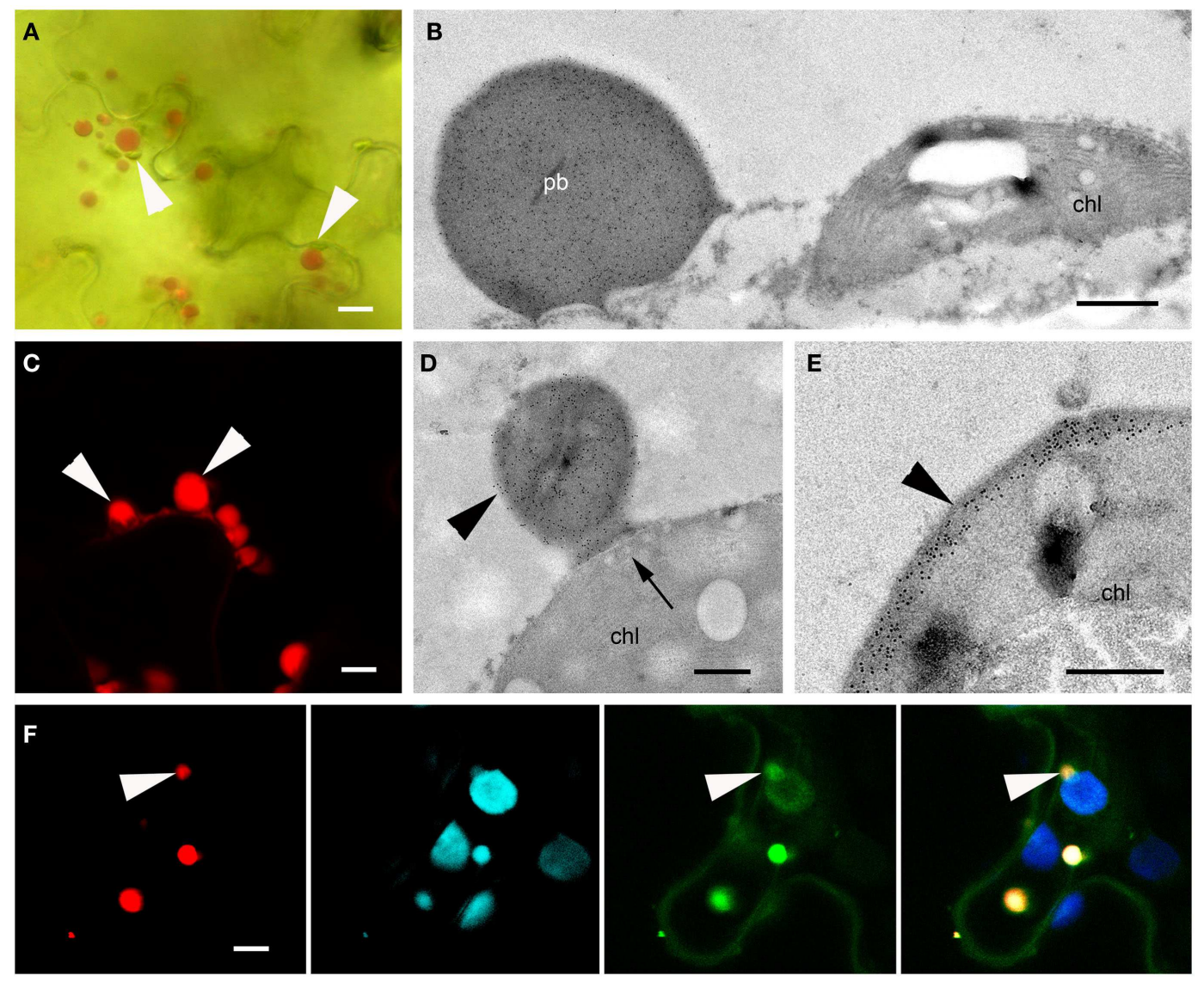

FIGURE 6 | Protein bodies induced by $\Delta$ SP-DsCRS. (A) Bright field microscopy. Several large fluorescent protein bodies can be observed, some in close association with a plastid (arrowhead). (B) Immunoelectron microscopy, localization of DsRed. Large protein body in the vicinity of a plastid (chl). (C) CLSM image of protein bodies budding off a plastid (arrowheads). (D,E) Immunoelectron microscopy, localization of the fluorescent fusion protein in the IMS. Protein body budding off a plastid
[(D), arrowhead, chl], showing tubular thylakoids close to the budding site [(D), arrow]. Abundant gold probes in the periphery of the plastid [(E), arrowhead]. (F) CLSM image of red fluorescent protein bodies enclosed by the outer plastid envelope membrane highlighted with a TOC-GFP membrane marker (arrowheads). DsRed (far left), plastid autofluorescence (middle-left), GFP (middle-right), and merged channels (far right) are shown. Bars $=10 \mu \mathrm{m}$ (A), $0.5 \mu \mathrm{m}$ (B,D-F), or $5 \mu \mathrm{m}$ (C).

\section{PB FORMATION IN THE INTERMEMBRANE SPACE}

The basal construct lacking an N-terminal signal peptide $(\triangle \mathrm{SP}$ DsZein) resulted in the unexpected formation of $\mathrm{PBs}$ associated with the plastid periphery. This distribution was clearly distinct from the stromal localization mediated by TP-DsZein, and electron microscopy duly confirmed that the protein was accumulating not in the stroma, but in the IMS of the plastid envelope. This was confirmed by transient expression in transgenic tobacco plants expressing a fluorescent marker protein highlighting the outer plastid envelope. The ectopic PBs containing DsRed were budding off from the envelope into the cytoplasm. Whereas SP-DsZein and TP-DsZein induced the formation of homogeneous PBs that were insoluble under non-reducing conditions, the PBs formed by $\Delta$ SP-DsZein varied considerably in size and shape, and a significant proportion of the recombinant protein was soluble under non-reducing conditions indicating that it was not assembled into a disulfide-bonded polymer. This may indicate that targeting of the protein to the plastids was only partial. Indeed, it was not clear whether all the observed PBs had originated by budding from chloroplasts and we therefore cannot exclude the possibility that further PB-like fluorescent structures had also formed directly in the cytosol. Alternatively, the partial recovery of DsRed by extraction with a non-reducing buffer could mean that part of the protein that enters the IMS fails to form insoluble polymers. The finding that the solubility of $\gamma$-zein in non-reducing conditions is inversely related to the number of Cys residues in the N-terminal region supports this assumption (Mainieri et al., 2014). Whereas redox processes in the stroma and thylakoid lumen are well characterized, the redox state of the IMS has not been studied in detail (Herrmann et al., 2009; Stengel et al., 2010). Redox machineries equivalent to those catalyzing the oxidation of proteins in the periplasmic space of bacteria or the IMS of mitochondria have not been identified in the IMS of plastids, although it is a topologically equivalent compartment. It has been suggested, however, that TOC12, a subunit of the translocon on the outer chloroplast membrane (TOC) complex in the plastid IMS, may be oxidized as part of the redox-dependent regulation of plastid protein import (Balsera et al., 2010). Once it has crossed 
the outer plastid membrane the DsZein fusion protein may therefore assemble into disulfide-linked, insoluble polymers that are no longer competent for further transport.

\section{A PLASTID-TARGETING SIGNAL IN $\boldsymbol{\gamma}$-zein}

It is important to note that in the absence of the added $\gamma$-zein(493) sequence the DsRed sequence we used did not accumulate in tobacco plastids and did not form PBs (Jach et al., 2001). This suggests that the $\mathrm{N}$-terminal $\gamma$-zein sequence is responsible for plastid targeting when fused to the C-terminus of DsRed. Chloroplast transit peptides are usually located at the $\mathrm{N}$-terminus and are removed upon protein translocation, however, imported proteins exist that do not have removed peptides and contain targeting information at different locations along the mature sequence (Miras et al., 2002). We therefore sought the core sequence responsible for conferring this property, i.e., a cryptic plastid-targeting signal within the $\gamma$-zein sequence. Plastid transit peptides usually contain a high proportion of hydrophobic and basic amino acids but few acidic residues (Zhang and Glaser, 2002). The $\gamma$-zein CRS extending from residues 51-93 shares these properties and appeared to be the most likely plastid-targeting sequence based on in silico analysis (Emanuelsson et al., 2000). Indeed, when this sequence was fused to DsRed lacking any further targeting information (construct $\triangle$ SP-CRS) we observed plastid targeting and the formation of fluorescent PBs in the IMS, just as we observed with the complete $\gamma$-zein $\mathrm{N}$-terminal sequence, indicating that the CRS is the relevant sequence part causing plastid targeting. It is possible, however, that unidentified sequence motifs in the DsRed polypeptide may additionally contribute to targeting of the fusion.

It is unclear whether the $\Delta \mathrm{SP}$-DsZein protein enters the plastid via the TOC/TIC complexes (Li and Chiu, 2010) or a noncanonical route (Armbruster et al., 2009). The TOC/TIC pathway involves hetero-oligomeric complexes in the inner and outer plastid membranes (Jarvis and Soll, 2002; Kessler and Schnell, 2009). Import via the TOC channel requires at least four different TOC proteins, all sharing conserved cysteine residues (Stengel et al., 2009). The formation and reduction of disulfide bonds between TOC proteins was shown to regulate this trafficking step (Kessler and Schnell, 2009), but there is no evidence for the formation of disulfide bonds between TOC components and incoming preproteins (Stengel et al., 2010). However, the cysteine residues in the $\gamma$-zein CRS are probably exposed and in a reduced form in the cytosol, so the formation of disulfide bridges with TOC components might also occur and prevent further transport through the TIC receptor. Instead, the protein would remain in the IMS, where apparently the conditions are appropriate to support the formation of PBs. In contrast, TP-DsZein is probably recognized by the IMS translocation complex and therefore further transported via the TIC channel (Figure 7). Interaction studies will be necessary to characterize the import pathways in more detail. It was not possible to regenerate stable transgenic plants transformed with the $\triangle$ SP-DsZein and TP-DsZein constructs (data not shown) suggesting that the formation of PBs in the plastids was not compatible with the regeneration of viable plants, perhaps because the PBs disturbed the sensitive redox equilibrium in the plastids (Wittenberg and Danon, 2008).

It was remarkable that PBs not only formed in the plastid IMS but also budded from the surface in a similar manner to the normal budding of prolamin bodies from the ER in cereal endosperm cells. This indicates that both the biogenesis and the budding of PBs are independent of ER-specific factors, providing insight into the formation of endogenous storage organelles in cereal seeds. Although the mechanism of PB biogenesis is not fully understood, two driving forces have been previously identified and attributed to the $\gamma$-zein $\mathrm{N}$-terminal sequence, i.e., the formation of inter-chain disulfide bonds between cysteine residues and lateral interactions involving the amphipathic repeats (LlopTous et al., 2010). Both elements are considered necessary, so it is perhaps surprising that the CRS (which includes four cysteine residues but only a small part of the amphipathic repeat region)
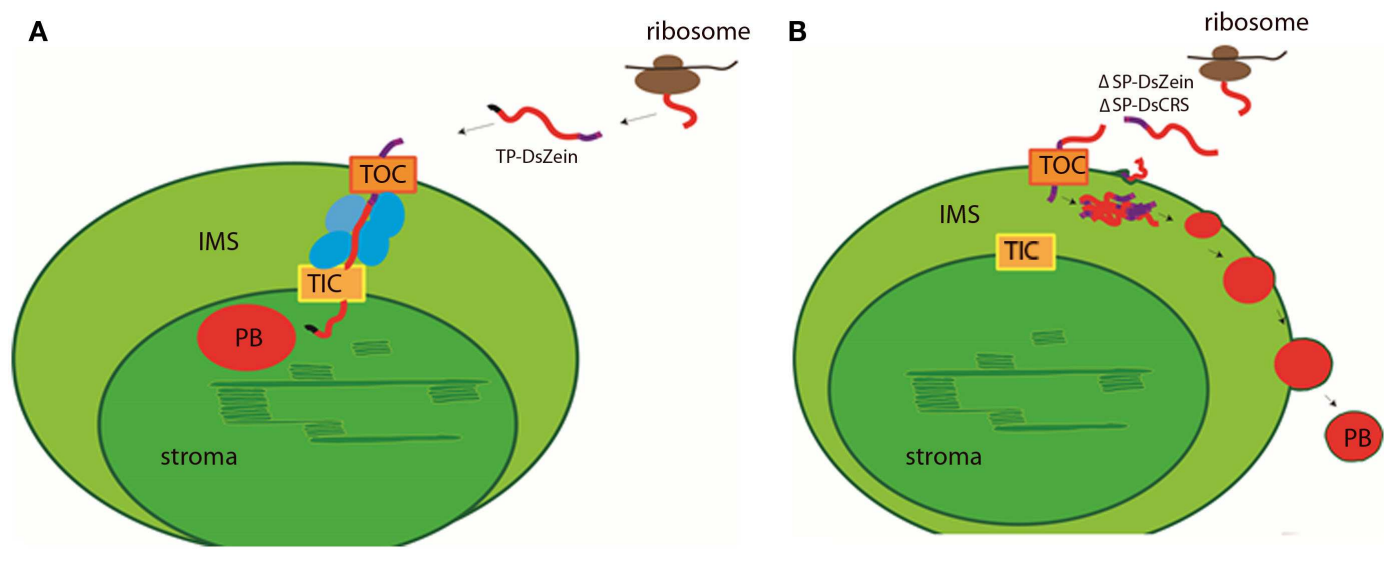

FIGURE 7 | Hypothetical model for the import of the fluorescent fusion proteins and for PB formation in the plastid. (A) TP-DsZein likely enters the plastid via the TOC/TIC pathway (orange). After passing the TOC complex, it is probably recognized by the IMS translocation complex (blue) and further transported via the TIC channel into the stroma where the transit peptide (black) is removed and a PB (red) is formed. (B) $\triangle$ SP-DsZein and $\triangle$ SP-DsCRS may enter the plastid via the TOC complex or a non-canonical route. After crossing the outer plastid membrane the fusion protein assembles into insoluble polymers that are no longer competent for further transport. PBs are formed in the IMS and bud off from the plastid. The DsRed sequence is shown in red, $\gamma$-zein sequences are shown in purple, ribosomes are marked in brown 
was sufficient to induce PB formation. This might be attributed to the design of our fusion construct, where tetrameric DsRed forms the N-terminal part and may support oligomerization, thus, functionally replacing the amphipathic repeats. The fusion partner has also been shown to influence PB biogenesis in previous studies. For example, phaseolin is a soluble homotrimer (Vitale et al., 1995) and induces efficient zeolin PB formation when fused to the $\gamma$-zein $\mathrm{N}$ terminal sequence (Mainieri et al., 2004). In contrast, PBs were not formed when the Human immunodeficiency virus Nef antigen was fused to the $\gamma$-zein $\mathrm{N}$-terminal sequence, whereas PB formation was possible when Nef was fused to the entire chimeric protein zeolin (De Virgilio et al., 2008).

\section{CONCLUDING REMARKS}

We have shown that PBs can be induced to form outside the endomembrane system, and we have identified a cryptic plastidtargeting signal in the $\mathrm{N}$-terminal portion of the $27 \mathrm{kDa} \gamma$-zein protein. We have shown that this cysteine-rich sequence mediates the transport of DsRed across the outer plastid envelope and can induce the formation of PBs in the plastid IMS. These results add to our knowledge on endogenous and ectopic $\mathrm{PB}$ formation by demonstrating that $\mathrm{PB}$ formation and budding do not require ERspecific factors. It will be interesting to attempt the formation of plastid-targeted PBs with other fusion proteins to determine the robustness of this strategy for recombinant protein production in transient expression systems.

\section{ACKNOWLEDGMENTS}

The authors would like to acknowledge financial support by the Austrian Science Fund FWF (W1224 and I1461-B16) and the "Filagro" project of CNR-Regione Lombardia.

\section{SUPPLEMENTARY MATERIAL}

The Supplementary Material for this article can be found online at http://www.frontiersin.org/Journal/10.3389/fbioe.2014.00067/ abstract

\section{REFERENCES}

Arcalis, E., Stadlmann, J., Marcel, S., Drakakaki, G., Winter, V., Rodriguez, J., et al. (2010). The changing fate of a secretory glycoprotein in developing maize endosperm. Plant Physiol. 153, 693-702. doi:10.1104/pp.109.152363

Armbruster, U., Hertle, A., Makarenko, E., Zuhlke, J., Pribil, M., Dietzmann, A., et al. (2009). Chloroplast proteins without cleavable transit peptides: rare exceptions or a major constituent of the chloroplast proteome? Mol. Plant. 2, 1325-1335. doi: $10.1093 / \mathrm{mp} / \mathrm{ssp} 082$

Bagga, S., Adams, H., Kemp, J. D., and Sengupta-Gopalan, C. (1995). Accumulation of 15-kilodalton zein in novel protein bodies in transgenic tobacco. Plant Physiol. 107, 13-23.

Bally, J., Paget, E., Droux, M., Job, C., Job, D., and Dubald, M. (2008). Both the stroma and thylakoid lumen of tobacco chloroplasts are competent for the formation of disulphide bonds in recombinant proteins. Plant Biotechnol. J. 6, 46-61. doi:10.1111/j.1467-7652.2007.00298.x

Balsera, M., Soll, J., and Buchanan, B. B. (2010). Redox extends its regulatory reach to chloroplast protein import. Trends Plant Sci. 15, 515-521. doi:10.1016/j.tplants. 2010.06.002

Bellucci, M., De Marchis, F., Mannucci, R., Bock, R., and Arcioni, S. (2005). Cytoplasm and chloroplasts are not suitable subcellular locations for beta-zein accumulation in transgenic plants. J. Exp. Bot. 56, 1205-1212. doi:10.1093/jxb/eri114

Bellucci, M., De Marchis, F., Nicoletti, I., and Arcioni, S. (2007). Zeolin is a recombinant storage protein with different solubility and stability properties according to its localization in the endoplasmic reticulum or in the chloroplast. J. Biotechnol. 131, 97-105. doi:10.1016/j.jbiotec.2007.06.004

Chikwamba, R. K., Scott, M. P., Mejia, L. B., Mason, H. S., and Wang, K. (2003). Localization of a bacterial protein in starch granules of transgenic maize kernels. Proc. Natl. Acad. Sci. U. S. A. 100, 11127-11132. doi:10.1073/pnas. 1836901100

Conley, A. J., Joensuu, J. J., Menassa, R., and Brandle, J. E. (2009). Induction of protein body formation in plant leaves by elastin-like polypeptide fusions. BMC Biol. 7:48. doi:10.1186/1741-7007-7-48

Daniell, H., Lee, S. B., Panchal, T., and Wiebe, P. O. (2001). Expression of the native cholera toxin B subunit gene and assembly as functional oligomers in transgenic tobacco chloroplasts. J. Mol. Biol. 311, 1001-1009. doi:10.1006/jmbi.2001.4921

De Marchis, F., Pompa, A., and Bellucci, M. (2012). Plastid proteostasis and heterologous protein accumulation in transplastomic plants. Plant Physiol. 160, 571-581. doi:10.1104/pp.112.203778

De Marchis, F., Pompa, A., Mannucci, R., Morosinotto, T., and Bellucci, M. (2011). A plant secretory signal peptide targets plastome-encoded recombinant proteins to the thylakoid membrane. Plant Mol. Biol. 76, 427-441. doi:10.1007/s11103010-9676-6

De Virgilio, M., De Marchis, F., Bellucci, M., Mainieri, D., Rossi, M., Benvenuto, E., et al. (2008). The human immunodeficiency virus antigen Nef forms protein bodies in leaves of transgenic tobacco when fused to zeolin. J. Exp. Bot. 59, 2815-2829. doi:10.1093/jxb/ern143

Emanuelsson, O., Nielsen, H., Brunak, S., and Von Heijne, G. (2000). Predicting subcellular localization of proteins based on their N-terminal amino acid sequence. J. Mol. Biol. 300, 1005-1016. doi:10.1006/jmbi.2000.3903

Floss, D. M., Sack, M., Stadlmann, J., Rademacher, T., Scheller, J., Stoger, E., et al. (2008). Biochemical and functional characterization of anti-HIV antibodyELP fusion proteins from transgenic plants. Plant Biotechnol. J. 6, 379-391. doi:10.1111/j.1467-7652.2008.00326.x

Geli, M. I., Torrent, M., and Ludevid, D. (1994). Two structural domains mediate two sequential events in [gamma]-zein targeting: protein endoplasmic reticulum retention and protein body formation. Plant Cell 6, 1911-1922. doi:10.2307/3869917

Glenz, K., Bouchon, B., Stehle, T., Wallich, R., Simon, M. M., and Warzecha, H. (2006). Production of a recombinant bacterial lipoprotein in higher plant chloroplasts. Nat. Biotechnol. 24, 76-77. doi:10.1038/nbt1170

Gutierrez, S. P., Saberianfar, R., Kohalmi, S. E., and Menassa, R. (2013). Protein body formation in stable transgenic tobacco expressing elastin-like polypeptide and hydrophobin fusion proteins. BMC Biotechnol. 13:40. doi:10.1186/1472-675013-40

Hanson, M. R., and Sattarzadeh, A. (2008). Dynamic morphology of plastids and stromules in angiosperm plants. Plant Cell Environ. 31, 646-657. doi:10.1111/j. 1365-3040.2007.01768.x

Herrmann, J. M., Kauff, F., and Neuhaus, H. E. (2009). Thiol oxidation in bacteria, mitochondria and chloroplasts: common principles but three unrelated machineries? Biochim. Biophys. Acta 1793, 71-77. doi:10.1016/j.bbamcr.2008. 05.001

Hofbauer, A., and Stoger, E. (2013). Subcellular accumulation and modification of pharmaceutical proteins in different plant tissues. Curr. Pharm. Des. 19, 5495-5502. doi:10.2174/1381612811319310005

Jach, G., Binot, E., Frings, S., Luxa, K., and Schell, J. (2001). Use of red fluorescent protein from Discosoma sp. (dsRED) as a reporter for plant gene expression. Plant J. 28, 483-491. doi:10.1046/j.1365-313X.2001.01153.x

Jarvis, P., and Soll, J. (2002). Toc, tic, and chloroplast protein import. Biochim. Biophys. Acta 1590, 177-189. doi:10.1016/S0167-4889(02)00176-3

Joseph, M., Ludevid, M. D., Torrent, M., Rofidal, V., Tauzin, M., Rossignol, M., et al. (2012). Proteomic characterisation of endoplasmic reticulum-derived protein bodies in tobacco leaves. BMC Plant Biol. 12:36. doi:10.1186/1471-2229-12-36

Kessler, F., and Schnell, D. (2009). Chloroplast biogenesis: diversity and regulation of the protein import apparatus. Curr. Opin. Cell Biol. 21, 494-500. doi:10.1016/j.ceb.2009.03.004

Lakshmi, P. S., Verma, D., Yang, X., Lloyd, B., and Daniell, H. (2013). Low cost tuberculosis vaccine antigens in capsules: expression in chloroplasts, bioencapsulation, stability and functional evaluation in vitro. PLoS ONE 8:e54708. doi:10.1371/journal.pone.0054708

Lau, E. T., Giddings, S. J., Mohammed, S. G., Dubois, P., Johnson, S. K., Stanley, R. A., et al. (2013). Encapsulation of hydrocortisone and mesalazine in zein microparticles. Pharmaceutics 5, 277-293. doi:10.3390/pharmaceutics5020277 
Lending, C. R., and Larkins, B. A. (1989). Changes in the zein composition of protein bodies during maize endosperm development. Plant Cell 1, 1011-1023. doi: $10.2307 / 3869002$

Li, H. M., and Chiu, C. C. (2010). Protein transport into chloroplasts. Annu. Rev. Plant Biol. 61, 157-180. doi:10.1146/annurev-arplant-042809-112222

Liu, X., Sun, Q., Wang, H., Zhang, L., and Wang, J. Y. (2005). Microspheres of corn protein, zein, for an ivermectin drug delivery system. Biomaterials 26, 109-115. doi:10.1016/j.biomaterials.2004.02.013

Llop-Tous, I., Madurga, S., Giralt, E., Marzabal, P., Torrent, M., and Ludevid, M. D. (2010). Relevant elements of a maize gamma-zein domain involved in protein body biogenesis. J. Biol. Chem. 285, 35633-35644. doi:10.1074/jbc.M110.116285

Llop-Tous, I., Ortiz, M., Torrent, M., and Ludevid, M. D. (2011). The expression of a xylanase targeted to ER-protein bodies provides a simple strategy to produce active insoluble enzyme polymers in tobacco plants. PLoS ONE 6:e19474. doi:10.1371/journal.pone.0019474

Ludevid, M. D., Torrent, M., Martinez-Izquierdo, J. A., Puigdomenech, P., and Palau, J. (1984). Subcellular localization of glutelin-2 in maize (Zea mays L.) endosperm. Plant Mol. Biol. 3, 227-234. doi:10.1007/BF00029658

Ma, J. K., Christou, P., Chikwamba, R., Haydon, H., Paul, M., Ferrer, M. P., et al. (2013). Realising the value of plant molecular pharming to benefit the poor in developing countries and emerging economies. Plant Biotechnol. J. 11, 1029-1033. doi:10.1111/pbi.12127

Mainieri, D., Morandini, F., Maîtrejean, M., Saccani, A., Pedrazzini E., and Vitale A. (2014). Protein body formation in the endoplasmic reticulum as an evolution of storage protein sorting to vacuoles: insights from maize $\gamma$-zein. Front. Plant Sci. 5:331. doi:10.3389/fpls.2014.00331

Mainieri, D., Rossi, M., Archinti, M., Bellucci, M., De Marchis, F., Vavassori, S., et al. (2004). Zeolin. A new recombinant storage protein constructed using maize $\gamma$-zein and bean phaseolin. Plant Physiol. 136, 3447-3456. doi:10.1104/pp.104. 046409

Miras, S., Salvi, D., Ferro, M., Grunwald, D., Garin, J., Joyard, J., et al. (2002). Noncanonical transit peptide for import into the chloroplast. J. Biol. Chem. 277, 47770-47778. doi:10.1074/jbc.M207477200

Monselise, E. B. I., Porath, D., and Tal, M. (1984). Unusual tubular clusters in the plastids of a duckweed (Lemna-Paucicostata) mutant incapable of photosynthesis and ammonium ion uptake. New Phytol. 98, 249-257. doi:10.1111/j.14698137.1984.tb02735.x

Nochi, T., Takagi, H., Yuki, Y., Yang, L., Masumura, T., Mejima, M., et al. (2007). Rice-based mucosal vaccine as a global strategy for cold-chain- and needle-free vaccination. Proc. Natl. Acad. Sci. U. S. A. 104, 10986-10991. doi:10.1073/pnas. 0703766104

Pompa, A., and Vitale, A. (2006). Retention of a bean phaseolin/maize gamma-zein fusion in the endoplasmic reticulum depends on disulfide bond formation. Plant Cell 18, 2608-2621. doi:10.1105/tpc.106.042226

Rybicki, E. P., Hitzeroth, I. I., Meyers, A., Dus Santos, M. J., and Wigdorovitz, A. (2013). Developing country applications of molecular farming: case studies in South Africa and Argentina. Curr. Pharm. Des. 19, 5612-5621. doi:10.2174/ 1381612811319310015

Shewry, P. R., and Halford, N. G. (2002). Cereal seed storage proteins: structures, properties and role in grain utilization. J. Exp. Bot. 53, 947-958. doi:10.1093/ jexbot/53.370.947

Shigemitsu, T., Masumura, T., Morita, S., and Satoh, S. (2013). Accumulation of rice prolamin-GFP fusion proteins induces ER-derived protein bodies in transgenic rice calli. Plant Cell Rep. 32, 389-399. doi:10.1007/s00299-012-1372-3

Staub, J. M., Garcia, B., Graves, J., Hajdukiewicz, P. T., Hunter, P., Nehra, N., et al. (2000). High-yield production of a human therapeutic protein in tobacco chloroplasts. Nat. Biotechnol. 18, 333-338. doi:10.1038/73796

Stengel, A., Benz, J. P., Buchanan, B. B., Soll, J., and Bölter, B. (2009). Preprotein import into chloroplasts via the Toc and Tic complexes is regulated by redox signals in Pisum sativum. Mol. Plant. 2, 1181-1197. doi:10.1093/mp/ssp043
Stengel, A., Benz, J. P., Soll, J., and Bolter, B. (2010). Redox-regulation of protein import into chloroplasts and mitochondria: similarities and differences. Plant Signal. Behav. 5, 105-109. doi:10.4161/psb.5.2.10525

Stoger, E., Fischer, R., Moloney, M., and Ma, J. K. (2014). Plant molecular pharming for the treatment of chronic and infectious diseases. Annu. Rev. Plant Biol. 65, 743-768. doi:10.1146/annurev-arplant-050213-035850

Takagi, H., Hiroi, T., Hirose, S., Yang, L., and Takaiwa, F. (2010). Rice seed ER-derived protein body as an efficient delivery vehicle for oral tolerogenic peptides. Peptides 31, 1421-1425. doi:10.1016/j.peptides.2010.04.032

Torrent, M., Llompart, B., Lasserre-Ramassamy, S., Llop-Tous, I., Bastida, M., Marzabal, P., et al. (2009a). Eukaryotic protein production in designed storage organelles. BMC Biol. 7:5. doi:10.1186/1741-7007-7-5

Torrent, M., Llop-Tous, I., and Ludevid, M. D. (2009b). Protein body induction: a new tool to produce and recover recombinant proteins in plants. Methods Mol. Biol. 483, 193-208. doi:10.1007/978-1-59745-407-0_11

Vitale, A., Bielli, A., and Ceriotti, A. (1995). The binding protein associates with monomeric phaseolin. Plant Physiol. 107, 1411-1418.

Wakasa, Y., Takagi, H., Hirose, S., Yang, L., Saeki, M., Nishimura, T., et al. (2013). Oral immunotherapy with transgenic rice seed containing destructed Japanese cedar pollen allergens, Cry j 1 and Cry j 2, against Japanese cedar pollinosis. Plant Biotechnol. J. 11, 66-76. doi:10.1111/pbi.12007

Wang, R., Tian, Z., and Chen, L. (2011). Nano-encapsulations liberated from barley protein microparticles for oral delivery of bioactive compounds. Int. J. Pharm. 406, 153-162. doi:10.1016/j.ijpharm.2010.12.039

Whitehead, M., Ohlschläger, P., Almajhdi, F. N., Alloza, L., Marzábal, P., Meyers, A. E., et al. (2014). Human papillomavirus (HPV) type 16 E7 protein bodies cause tumour regression in mice. BMC Cancer 14:367. doi:10.1186/1471-2407-14-367

Wittenberg, G., and Danon, A. (2008). Disulfide bond formation in chloroplasts: formation of disulfide bonds in signaling chloroplast proteins. Plant Sci. 175, 459-466. doi:10.1016/j.plantsci.2008.05.011

Zhang, X. P., and Glaser, E. (2002). Interaction of plant mitochondrial and chloroplast signal peptides with the Hsp70 molecular chaperone. Trends Plant Sci. 7, 14-21. doi:10.1016/S1360-1385(01)02180-X

Zhong, Q., and Jin, M. (2009). Nanoscalar structures of spray-dried zein microcapsules and in vitro release kinetics of the encapsulated lysozyme as affected by formulations. J. Agric. Food Chem. 57, 3886-3894. doi:10.1021/jf803951a

Zou, T., and Gu, L. (2013). TPGS emulsified zein nanoparticles enhanced oral bioavailability of daidzin: in vitro characteristics and in vivo performance. Mol. Pharm. 10, 2062-2070. doi:10.1021/mp400086n

Conflict of Interest Statement: The authors declare that the research was conducted in the absence of any commercial or financial relationships that could be construed as a potential conflict of interest.

Received: 15 October 2014; paper pending published: 04 November 2014; accepted: 24 November 2014; published online: 11 December 2014.

Citation: Hofbauer A, Peters J, Arcalis E, Rademacher T, Lampel J, Eudes F, Vitale $A$ and Stoger $E$ (2014) The induction of recombinant protein bodies in different subcellular compartments reveals a cryptic plastid-targeting signal in the 27-kDa $\gamma$-zein sequence. Front. Bioeng. Biotechnol. 2:67. doi: 10.3389/fbioe.2014.00067

This article was submitted to Plant Biotechnology, a section of the journal Frontiers in Bioengineering and Biotechnology.

Copyright (C) 2014 Hofbauer, Peters, Arcalis, Rademacher, Lampel, Eudes, Vitale and Stoger. This is an open-access article distributed under the terms of the Creative Commons Attribution License (CC BY). The use, distribution or reproduction in other forums is permitted, provided the original author(s) or licensor are credited and that the original publication in this journal is cited, in accordance with accepted academic practice. No use, distribution or reproduction is permitted which does not comply with these terms. 